\title{
The Role of Telemedicine in Surgical Specialties During the COVID-19 Pandemic: A Scoping Review
}

\author{
Mahir Gachabayov ${ }^{1,3} \cdot$ Lulejeta A. Latifi ${ }^{1,2,3} \cdot$ Afshin Parsikia $^{1,3} \cdot$ Rifat Latifi $^{1,3}$
}

Accepted: 7 October 2021 / Published online: 6 November 2021

(C) Société Internationale de Chirurgie 2021

\begin{abstract}
Background The objective of this study was to evaluate the current body of evidence on the use of telemedicine in surgical subspecialties during the COVID-19 pandemic.

Methods This was a scoping review conducted in compliance with the Preferred Reporting Items for Systematic Reviews and Meta-Analyses extension for scoping reviews (PRISMA-ScR). MEDLINE via Ovid, PubMed, and EMBASE were systematically searched for any reports discussing telemedicine use in surgery and surgical specialties during the first period (February 2020-August 8, 2020) and second 6-month period (August 9-March 4, 2021) of the COVID-19 pandemic.

Results Of 466 articles screened through full text, 277 articles were included for possible qualitative and/or quantitative data synthesis. The majority of publications in the first 6 months were in orthopedic surgery, followed by general surgery and neurosurgery, whereas in the second 6 months of COVID-19 pandemic, urology and neurosurgery were the most productive, followed by transplant and plastic surgery. Most publications in the first 6 months were opinion papers (80\%), which decreased to $33 \%$ in the second 6 months. The role of telemedicine in different aspects of surgical care and surgical education was summarized stratifying by specialty.

Conclusion Telemedicine has increased access to care of surgical patients during the COVID-19 pandemic, but whether this practice will continue post-pandemic remains unknown.
\end{abstract}

\section{Introduction}

The first case of unknown pneumonia was reported in Wuhan City, Hubei Province of China, on December 31, 2019. After only 71 days on March 11, 2020, the World

Rifat Latifi

Rifat.Latifi@wmchealth.org

1 Department of Surgery, Westchester Medical Center Health, New York Medical College, School of Medicine, Taylor Pavilion, Suite D334, 100 Woods Road, Valhalla, NY 10595, USA

2 Present Address: University of Arizona, Tucson, AZ, USA

3 Department of Surgery, Westchester Medical Center, New York Medical College, Valhalla, NY, USA
Health Organization declared the COVID-19 pandemic, historically the first caused by a coronavirus. The first case in the USA was reported on January 11, 2020. Globally, as of July 21, 2021, there have been 191,148,056 confirmed cases of COVID-19, including 4,109,303 deaths, as reported by WHO [1]. As of July 20, 2021, a total of $3,568,861,733$ vaccine doses have been administered. In the USA alone as of July 22, 2021, >600,000 people have died [2].

Overall, the COVID-19 pandemic has caused major disruption, but it is surgical practice that has suffered the most. A moratorium on elective procedures was implemented in the majority of hospitals [3-5]. Deferral of elective care has increased complications in many types of surgical diseases, especially in patients with cancers, 
recurrent inflammatory diseases, and/or chronic organ failures [6]. The need for mandatory physical distancing and the fear of transmission of the virus during COVID-19 increased the role of telemedicine [7,8]. The objective of this study was to review the current body of evidence and the implementation of telemedicine in surgical subspecialties during the COVID-19 pandemic.

\section{Methods}

\section{Design and research question}

The protocol of this scoping review was developed and published prospectively [9] and complies with prior developed protocol as well as the Preferred Reporting Items for Systematic Reviews and Meta-Analyses (PRISMA) extension for scoping reviews (PRISMA-ScR) [10]. Three independent researchers carried out the literature search followed by screening of the yielded records (MG, AP, and LAL). The quality assessment of the included studies was carried out by two researchers independently (MG and AP) with the assistance of the senior author (RL). Three independent researchers (MG, AP, and LAL) performed data extraction and analysis followed by critical appraisal; any disagreements were resolved after discussion with the senior author (RL). The research question was formulated within the PICOTS framework as follows:

(P) Population Any reports discussing telemedicine regardless of the design, methodological quality, or level of evidence.

(I) Intervention Use of telemedicine.

(C) Comparator intervention Not applicable.

(O) Outcomes Description of study and future perspectives.

(T) Time Short- or long-term.

(S) Setting In- or outpatient.

\section{Eligibility criteria and endpoints}

The inclusion criterion was any reports discussing telemedicine use in surgical specialties during the first and second 6 months of the COVID-19 pandemic regardless of the design, methodological quality, or level of evidence. Exclusion criterion was lack of report of the outcomes of interest. The endpoints of interest were current state of implementation, future perspectives of telemedicine in surgery, and surgical subspecialties during the COVID-19 pandemic.

\section{Search strategy and study selection}

MEDLINE via Ovid, PubMed, and EMBASE were systematically searched for papers published in English with the following terms: 'telemedicine', 'telehealth', 'ehealth', 'virtual', 'video', 'digital divide', 'remote consultation', 'Covid-19', and 'surgery'. The Medical Subject Heading terms for surgical specialties were included in the advanced search as well. In addition, ClinicalTrials.gov was searched for any ongoing studies. Moreover, medRxiv.org was searched for unpublished studies and gray literature. Relevant records were identified, and the records yielded from the literature search were screened through by title, abstract, and/or full-text article. References of the included articles were screened to test the sensitivity of the search for additional publications.

\section{Data extraction and quality assessment}

Data from included studies were extracted and collected to prospectively design MS Excel tables by two independent reviewers (MG and AP). Collected data included author, publication, country, study design, and outcomes of interest. The quality assessment of the included studies was carried out using Oxford Centers for Evidence-Based Medicine classification of levels of evidence [11].

\section{Data analysis and presentation}

Extracted data from the included papers to predefined tables were formulated with standardized concepts to ensure that the findings were generalizable and to address the predefined objectives and research questions. A combination of an inductive and deductive approach was implemented for iterative process and qualitative data analysis. Standardized concepts examined by this scoping review aimed to clearly guide the scope and breadth of the inquiry. Concepts relevant to the current implementation of telemedicine, challenges that it faces, and future perspectives of its use and development were mostly generated using inductive approach. They were discussed between the three independent researchers (MG, LAL, and AP) in the process of data extraction, and any discrepancies were resolved in discussion with the senior author. Qualitative and quantitative data syntheses were performed using Microsoft Excel (Microsoft; Redmond, WA). Tables, histograms, pie charts, arrow plots, and radial graphs were used to present the findings of this scoping review. The graphs were generated using DataWrapper.de (Datawrapper; Berlin, Germany) in addition to Microsoft Excel. 


\section{Results}

The details of the study selection process are depicted in Fig. 1. A total of 3001 articles were yielded by the search of the three databases, and 1810 of those were screened through title and abstract by three independent reviewers (MG, AP, and LAL). After excluding non-relevant records, 466 articles were screened through full texts. Ultimately, 277 articles were included for possible qualitative and/or quantitative data synthesis. The search of the databases for the first and second six months was performed on August 8, 2020, and March 4, 2021, respectively.

A search revealed a twofold increase in publications searchable with the term 'telemedicine,' and more than a twofold increase in publications searchable with the terms 'telemedicine' and 'surgery' in 2020 as compared to 2019 (Fig. 2).

\section{Comparison of the first and second 6 months}

The vast majority of the articles on the use of telemedicine in surgical subspecialties during COVID-19 were published in the first six months (205 vs. 72 publications).

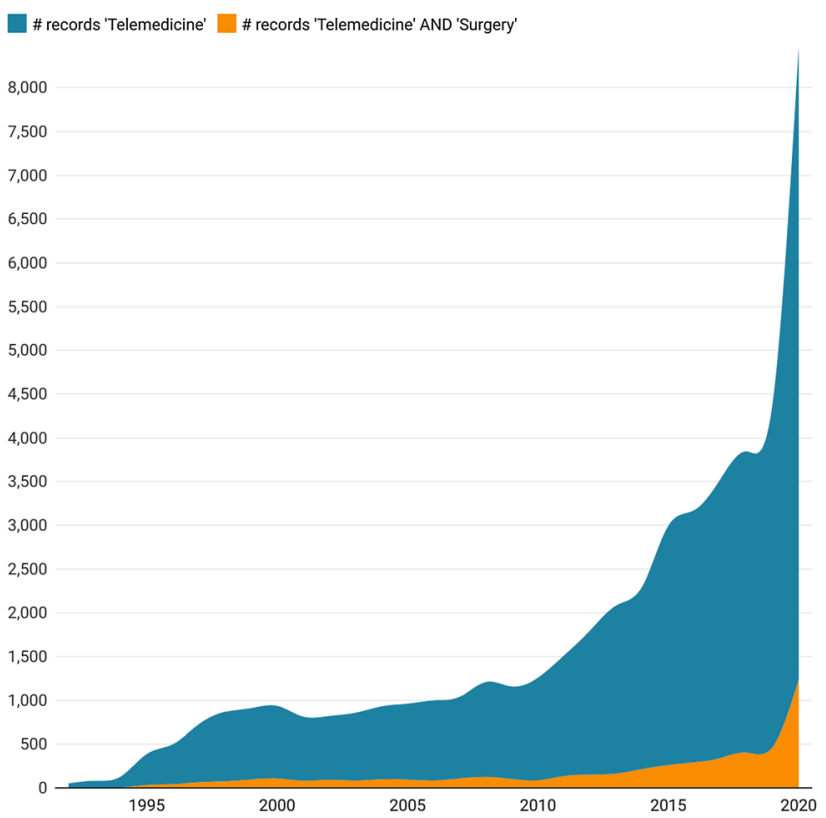

Fig. 2 Trend in publications on telemedicine

Figure 3 depicts the stratification of such articles by surgical specialty while comparing the first and second 6

Fig. 1 PRISMA flow diagram

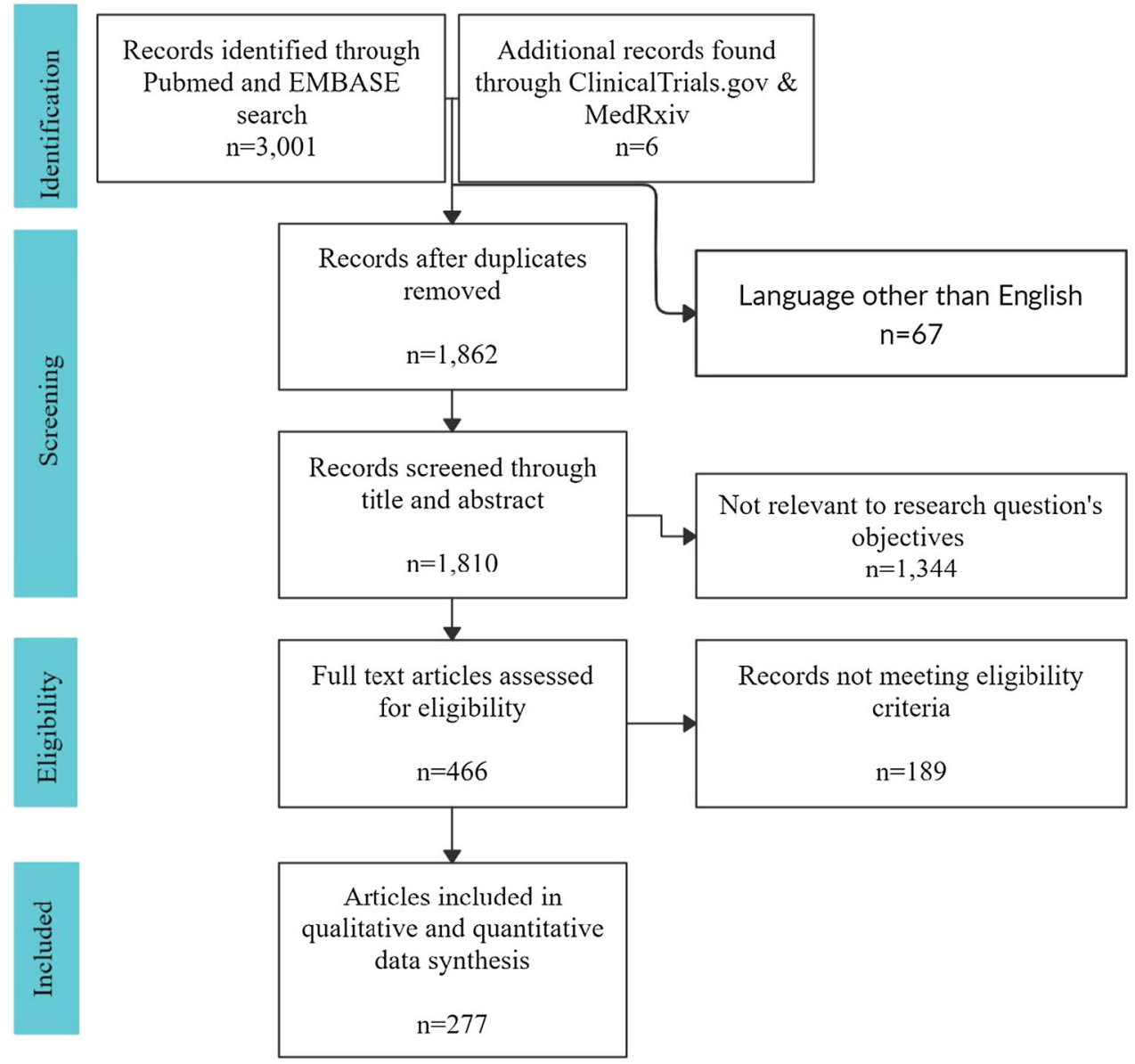




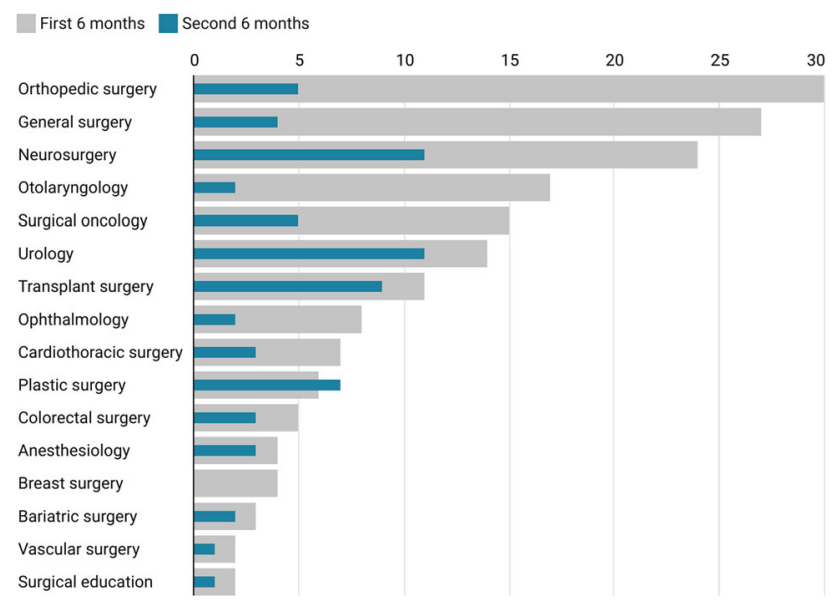

Fig. 3 Number of publications stratified by surgical specialty

months. It is noteworthy that the largest number of publications in the first 6 months was in orthopedic surgery followed by general surgery and neurosurgery, whereas in the second 6 months, urology and neurosurgery were the most productive, followed by transplant and plastic surgery.

Another important difference between the first and second 6 months was found in the types of articles (Fig. 4). In fact, the overwhelming majority of publications in the first 6 months were opinion articles $(80 \%)$, followed by summary design studies (9\%), cross-sectional surveys $(4 \%)$, clinical case or data reports $(4 \%)$, and guidelines or position statements (3\%). In the second 6 months, this distribution changed drastically so that the proportion of opinion articles decreased to $33 \%$, whereas the proportion of summary design studies, cross-sectional surveys, clinical case or data reports, and guidelines or position statements increased to $12 \%, 25 \%, 24 \%$, and $6 \%$, respectively.

\section{Telemedicine in surgery and surgical specialties during the pandemic}

The phases and the reasons for the use of telemedicine in surgical specialties (general surgery, bariatric surgery, surgical oncology, cardiac surgery, colorectal surgery, head and neck surgery, transplant surgery, surgical education, plastic surgery, neurosurgery, ophthalmology, otolaryngology, orthopedic surgery, and urology) are generalized and summarized in Fig. 5. Selected publications among included records are presented in Supplemental Table 1.

Many authors advocated for the active use of telemedicine clinics in general surgery during the first six months of the pandemic [12], which was believed to play a key role in the perioperative management of surgical patients during COVID-19 pandemic, allowing monitoring progression of the underlying disease and determining urgency [13]. In a cross-sectional survey, $88.5 \%$ of surgeons and $91 \%$ of patients reported positive perceptions on the use of telemedicine [14]. In another cross-sectional survey, where $86 \%$ of respondents were somewhat or extremely satisfied with telemedicine encounters, only onethird, nonetheless, preferred telemedicine after COVIDrelated social distancing ends [15]. The two-thirds preferring actual visits felt that "establishing trust and comfort" was best accomplished in person.

Telemedicine in postoperative care and follow-up of bariatric patients has been suggested as an important modality of communications [16].

While the use of telemedicine has shown different diffusion into clinical practice in different countries [17], there were few challenges. In this study, from Italy the authors reported a $24 \%$ no-show rate with the implementation of telemedicine seemingly due to technology-related factors as well as lack of digital literacy [18]. On the other hand, another study conducted at a well-established
Fig. 4 Types of articles published in the first and second 6 months

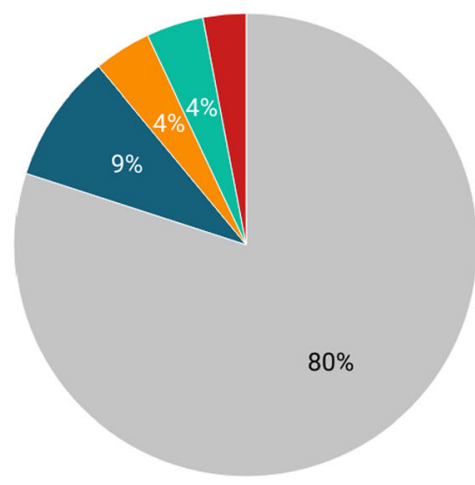

First 6 months

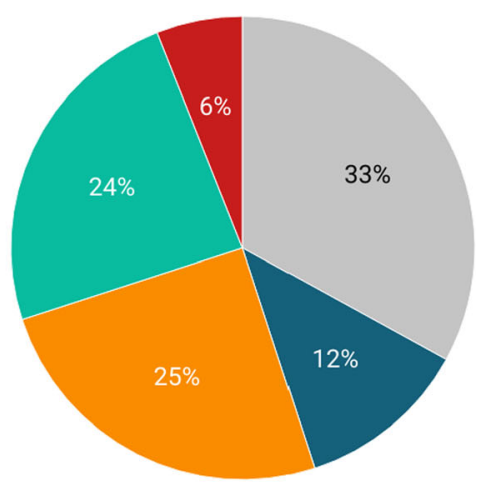

Second 6 months 


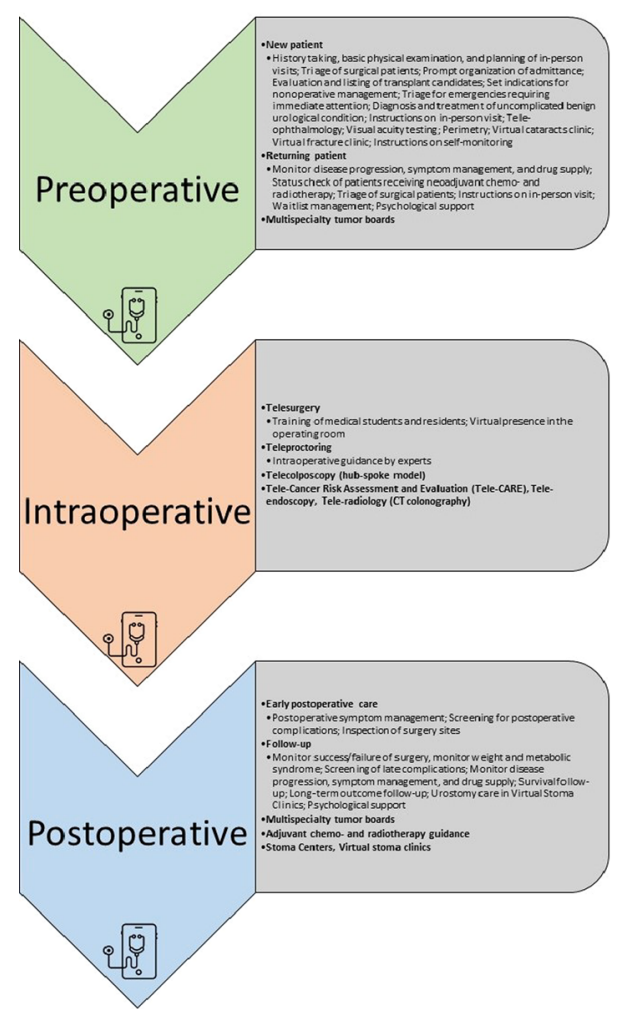

Fig. 5 Implementation of telemedicine in surgery and surgical specialties

comprehensive bariatric center found the no-show rate to be decreasing in the post-pandemic era [19].

Active use of telemedicine in oncology patients has been reported for remote management of chemotherapy, symptom management, overall supervision of care, palliative care, and clinical trials [20]. A 3-tiered approach: high-, medium-, and low-priority for telemedicine in patients with pancreatic cancer was reported [21], while other reported use of telemedicine for disease progression or recurrence breast cancer [22, 23], as well as assessment of signs of signs and symptoms of COVID-19 before surgery [24]. In the second 6 months of the pandemic, position statements of different societies have also found benefits of telemedicine in preoperative assessment and triage, as well as postoperative follow-up in patients with gynecological cancers $[25,26]$, particularly in large screening programs [27], and colorectal surgery [28, 29].

Telemedicine has been used in cardiac surgery for cases in which physical examination was not required prior to the pandemic [30, 31]. In addition, telemedicine was also reported to be associated with high provider and patient satisfaction rates in aortic disease for first evaluations and routine surveillance, but not for routine postoperative visits [32]. Store-and-forward telemedicine is thought to enhance the utility of real-time consults and decrease the costs of ambulatory clinic visits [33]. Another benefit of telemedicine was intraoperative teleproctoring while performing challenging procedures [34]. Need for increased knowledge and skills on the use of technology, payment system, credentialing for effective implementation of telemedicine, as well as understanding the modalities of telemedicine were identified as challenges [35].

Patients undergoing head and neck surgery were generally highly satisfied with their telemedicine experience [36]. Telemedicine was utilized in doctor-nurse consultations, outpatient wound care, home-based palliative care, academic meetings, and tumor board conferences [37]. Patient selection was considered one of the keys to success.

Telemedicine was advocated for liver transplant programs to expedite evaluation and listing of liver transplant candidates, improve readmission rates and quality of life, in addition to multidisciplinary virtual tumor boards for hepatocellular carcinoma cases [38]. One of the crosssectional studies evaluating current telemedicine use in the USA reported an increase of the telemedicine use rate from $16 \%$ in the pre-pandemic era to $98 \%$ [39]. Tele-hepatology program allows liver transplant evaluations and better triage, thereby decreasing the interval from referral to evaluation and listing [40-42], and improves patient compliance [42].

Despite the lack of widespread integration of telemedicine into the curricula in undergraduate medical education, medical students were able to complete virtual rotations, which allowed for participation in interactive live-streamed surgeries, outpatient telehealth visits, and virtual small group didactics during the pandemic [43]. In addition to creation of virtual consult rooms [44], weekly lectures, grand rounds, tumor boards, case conferences, journal clubs, and other similar academic and educational activities became online [45, 46].

The rate of telemedicine use in plastic surgery increased significantly from 23 to $91 \%$ during the pandemic [45] and afterward [46-48]. In one survey of plastic surgeons, $71 \%$ of the respondents stated that they plan to incorporate telemedicine in their daily practice even after the pandemic subsides. Patient satisfaction was equal to or better than face-to-face visits owing to decreased costs and time spent on travel [49].

In neurosurgical practice, the patient and provider satisfaction rates were reported to be $90 \%$ and $95 \%$, respectively [50]. The patient satisfaction rate was found to be even higher at $98 \%$ in a cross-sectional survey conducted in the Philippines [51]. A quite exemplary picture was described in a retrospective study comparing pre-pandemic 7 weeks to the 7 weeks following the start of the pandemic, which found an increase in the median number of telemedicine visits per week from 0 (IQR $0-0$ ) per week to 151 (IQR 126-156; $H=73.50 ; P<0.001$ ) [52]. A systematic review of 52 studies from the USA and international 
institutions found that telemedicine was successful in nearly $100 \%$ of the cases [53]. The rate of failure was less than $0.5 \%$, mostly related to technology and a need for face-to-face evaluation. In some institution, new and follow-up appointments were converted to telemedicine [54], while others offered patients and providers the choice between in-person and telemedicine visits [55], while others used it for effective remote proctoring during complex aneurysm cases [56].

In otolaryngology, telemedicine was especially helpful for patients with nasal symptoms, hearing impairment, tinnitus, treated sleep apnea, benign hoarseness, dizziness, and following simple plastic surgery [57]. In a survey evaluating the acceptability of telemedicine in oral and maxillofacial surgery in the UK, patients were found to be mostly happy with telemedicine [58]. However, inability to inspect cavities due to poor lighting during the telemedicine encounters [59, 60] was reported as one of the challenges.

In ophthalmologic practice, mobile applications were recommended for visual acuity testing and perimetry [61], as well as for prescription refills, medication delivery, and to teach self-monitoring [62], cataract service, and virtual glaucoma clinic [63]. Patients with ptosis, eyelid retraction, entropion, ectropion, dermatochalasis, epiphora, congenital deformities, and other conditions that rarely threaten vision can be identified readily using telemedicine [64].

In orthopedic surgery, face-to-face contact was recommended only for urgent cases and those cases when care cannot be delivered remotely including wound care, suture removal, evaluation for reduction of fractured bones, healing complications, and follow-up visits that change the treatment plan virtual fracture clinics [65]. Other reports indicated an increased rate of telemedicine use in orthopedic surgery [66-69]. A systematic review evaluating effectiveness and clinical relevance of virtual fracture clinics concluded that there was excellent evidence to support virtual fracture clinic for non-operative management of fifth metatarsal fractures, with moderate evidence for radial head and neck fractures [70]. Nonetheless, the routine use of virtual fracture clinics is presently not validated for all stable, undisplaced fracture patterns.

The adoption of telemedicine became more active in the second six months in urological practice, in particular for kidney stones [71]. In addition, a cross-sectional survey from the UK reported that a total of 2361 outpatient clinic (52.6\% for oncology and $47.4 \%$ benign urology) were scheduled. Of the total 1242 patients, $66.3 \%$ with oncology conditions were virtual consultations, $20 \%$ were face-toface, and $13.6 \%$ were canceled. Of the total benign outpatient conditions $(n=1119), 81 \%$ were virtual consultations, $9.7 \%$ were face-to-face, and $9.3 \%$ were canceled.
These findings indicate that telemedicine and telehealth should be whenever possible [72]].

\section{Discussion}

\section{Interpretation of the findings}

This scoping review evaluated and summarized the current literature on the actual and/or proposed the implementation of telemedicine in surgery and surgical subspecialties during the COVID-19 pandemic. The majority of the published papers in the first six months were expert opinions. In most cases, these opinions were based on the authors' experience of using telemedicine or proposing for a novel program (rarely) or re-exploring previous ideas. One of the most important findings of this scoping review was that the COVID-19 pandemic transformed telemedicine from an alternative to standard of care, used by a number of telemedicine enthusiast and institutions, to an essential modality of providing medical and surgical care, overcoming this way a well-known Semmelweis reflex.

Interestingly, there are substantial differences in the literature between the first and second six months since the onset of the COVID-19 pandemic. The second 6 months showed a clear shift from opinion-based articles to evidence-based reports. Secondly, the productivity of different surgical subspecialties differed between the evaluated periods. Although an increase was observed in almost all aspects of patients care, the mention of the use of telemedicine in preoperative decision making and survival follow-up slightly decreased. On the other hand, there was a large increase in outpatient management (virtual clinics), new patient consultation, telesurgery use in education, followed by preoperative evaluation/triage.

\section{Advantages of telemedicine}

Among others, telemedicine's major advantages include remote consultations, remote evaluation and management of surgical diseases, monitoring disease progress and drug supply, delivery of instructions, preoperative triage, immediate postoperative and/or long-term follow-ups, and multiple types of educational activities including telesurgery. The rate of satisfaction with telemedicine was estimated to be virtually maximal.

\section{Current challenges}

The challenges to a greater adoption and continued diffusion of telemedicine into our current daily practice were clearly formulated in the included expert opinions. The main challenges identified were jurisdictional boundaries, 
cross-state bounders practice, reimbursement, malpractice, and HIPAA protection. Other challenges include access to technology, particularly amongst elderly population leaving in rural settings, and digital literacy, as well as evaluation of patients with impaired vision or hearing or with other special needs. This challenge is another factor to be considered in digital "clinics" or telemedicine protocols.

Lastly, despite constantly growing telehealth and telementoring infrastructure and integration into the practice, most experts agreed that there is still no clear definition of the components of telemedicine and no clear patient selection criteria.

\section{Strengths and limitations}

The major strength of this scoping review was a rigorous literature search in addition to the large number of records included. Moreover, prospective development and publication of the protocol of this scoping review was another strength, which allowed minimizing reporting bias risks.

The biggest limitation of this scoping review was that all included records were either expert opinions with the level of evidence of 4 or cross-sectional surveys with the level of evidence of $3 b$. Another limitation was the fact that the research question of this scoping review was not the aim of most included papers but rather was reported as a secondary subject. This fact has probably led to a gross underreporting of the authors' experiences with telemedicine. An additional limitation is the fact that paper publication date or month may not be an accurate reflection of the time it was written and/or submitted, given the substantial heterogeneity in publication turnaround among medical journals.

\section{Conclusion}

Telemedicine has not only provided an adequate aid in the management of surgical patients during the moratorium of elective care imposed by the COVID-19 pandemic, but also was able to change the established long-standing surgical practice. Although the current body of evidence on the performance and clinical outcomes of telemedicine use in surgery is improving, more active research in different settings is needed. Moreover, there is need for an expert consensus statement in order to standardize patient selection criteria and a core descriptor set to guide future research.

Supplementary Information The online version contains supplementary material available at https://doi.org/10.1007/s00268021-06348-1.
Funding None.

\section{Declarations}

Conflict of interest The authors declared that they have no conflict of interest.

\section{References}

1. WHO Coronavirus (COVID-19) (2021) Dashboard [Internet]. Available from: https://covid19.who.int

2. Centers for Disease Control and Prevention (2021) COVID Data tracker [Internet]. Available from: https://covid.cdc.gov/coviddata-tracker/\#trends_dailytrendsdeaths

3. American College of Surgeons (ACS) (2020) COVID-19 update: guidance for triage of non-emergent surgical procedures. March 13, 2020. [Internet]. Available from: https://www.facs.org/covid19/clinical-guidance/triage

4. Zakka K, Erridge S, Chidambaram S, Beatty JW, Kynoch M, Kinross $\mathbf{J}$ et al (2020) Electrocautery, diathermy, and surgical energy devices: are surgical teams at risk during the COVID-19 pandemic? Ann Surg 272(3):e257-e262

5. Felsenreich DM, Gachabayov M, Dong XD, Cianchi F, Bergamaschi R (2020) Considerations on robotic colorectal surgery during a COVID-19 pandemic. Minerva Chir 75(4):213-215

6. Gachabayov M, Dong XD, Latifi R, Bergamaschi R (2020) Considerations on colorectal cancer care in a COVID-19 pandemic epicenter. Surg Technol Int 28(36):148-149

7. Latifi R (2020) Telehealth and the future of surgery. J Am Coll Surg 231(6):702-703

8. Latifi R, Doarn CR (2020) Perspective on COVID-19: finally, telemedicine at center stage. Telemed J E Health 26(9):1106-1109

9. Gachabayov M, Latifi LA, Parsikia A, Latifi R (2020) Current state and future perspectives of telemedicine use in surgery during the COVID-19 pandemic: a scoping review protocol. Int $\mathrm{J}$ Surg Protoc 24:17-20

10. Tricco AC, Lillie E, Zarin W, O'Brien KK, Colquhoun H, Levac $D$ et al (2018) PRISMA extension for scoping reviews (PRISMAScR): checklist and explanation. Ann Intern Med 169(7):467-473

11. OCEBM Levels of Evidence Working Group (2021) The Oxford 2011 Levels of Evidence. [Internet]. Available from: http://www. cebm.net/index.aspx?o=5653

12. Al-Jabir A, Kerwan A, Nicola M, Alsafi Z, Khan M, Sohrabi C et al (2020) Impact of the coronavirus (COVID-19) pandemic on surgical practice: part 1 . Int J Surg 79:168-179

13. Billig JI, Sears ED (2020) The compounding access problem for surgical care: innovations in the post-COVID era. Ann Surg 272(2):e47-e48

14. Zhu C, Williamson J, Lin A, Bush K, Hakim A, Upadhyaya K et al (2020) Implications for telemedicine for surgery patients after COVID-19: survey of patient and provider experiences. Am Surg 86(8):907-915

15. Sorensen MJ, Bessen S, Danford J, Fleischer C, Wong SL (2020) Telemedicine for surgical consultations: pandemic response or here to stay?: A report of public perceptions. Ann Surg 272(3):e174-e180

16. Walędziak M, Różańska-Walędziak A, Pędziwiatr M, Szeliga J, Proczko-Stepaniak M, Wysocki M et al (2020) Bariatric surgery during COVID-19 pandemic from patients' point of view-the results of a national survey. J Clin Med 9(6):E1697 
17. Beskow AF, Martinez-Duartez PR, Behrens Estrada EJ, Fiolo FE, Ramos AC (2020) CoViD-19 pandemic and bariatric surgery in Argentina. Obes Surg 30(12):5170-5176

18. Runfola M, Fantola G, Pintus S, Iafrancesco M, Moroni R (2020) Telemedicine implementation on a bariatric outpatient clinic during COVID-19 pandemic in Italy: an unexpected hill-start. Obes Surg 30(12):5145-5149

19. Brown AM, Ardila-Gatas J, Yuan V, Devas N, Docimo S, Spaniolas K et al (2020) The Impact of telemedicine adoption on a multidisciplinary bariatric surgery practice during the COVID19 pandemic. Ann Surg 272(6):e306-e310

20. Alshamrani M, AlHarbi A, Alkhudair N, AlNajjar F, Khan M, Obaid $A B$ et al (2020) Practical strategies to manage cancer patients during the COVID-19 pandemic: saudi oncology pharmacy assembly experts recommendations. J Oncol Pharm Pract 26(6):1429-1440

21. Catanese S, Pentheroudakis G, Douillard J-Y, Lordick F (2020) ESMO Management and treatment adapted recommendations in the COVID-19 era: pancreatic cancer. ESMO Open 5(Suppl 3): $\mathrm{e} 000804$

22. Viale G, Licata L, Sica L, Zambelli S, Zucchinelli P, Rognone A et al (2020) Personalized risk-benefit ratio adaptation of breast cancer care at the epicenter of COVID-19 outbreak. Oncologist 25(7):e1013-e1020

23. de Azambuja E, Trapani D, Loibl S, Delaloge S, Senkus E, Criscitiello $\mathrm{C}$ et al (2020) ESMO management and treatment adapted recommendations in the COVID-19 era: breast cancer. ESMO Open 5(Suppl 3):e000793

24. Fregatti P, Gipponi M, Giacchino M, Sparavigna M, Murelli F, Toni ML et al (2020) Breast cancer surgery during the COVID-19 pandemic: an observational clinical study of the breast surgery clinic at ospedale policlinico San Martino: Genoa. Italy In Vivo 34(3 Suppl):1667-1673

25. Ciavattini A, Delli Carpini G, Giannella L, Arbyn M, Kyrgiou M, Joura EA et al (2020) European federation for colposcopy (EFC) and European society of gynaecological oncology (ESGO) joint considerations about human papillomavirus (HPV) vaccination, screening programs, colposcopy, and surgery during and after the COVID-19 pandemic. Int J Gynecol Cancer 30(8):1097-1100

26. Lavoué V, Akladios C, Gladieff L, Classe J-M, Lécuru F, Collinet P (2020) Onco-gynecologic surgery in the COVID-19 era: risks and precautions-A position paper from FRANCOGYN, SCGP, SFCO, and SFOG. J Gynecol Obstet Hum Reprod. 49(7): 101787

27. Sterpetti AV (2020) Surgical oncology in the pandemic. lessons learned and future perspectives. Eur J Surg Oncol. 46(11):2162-2163

28. Pata F, Bondurri A, Ferrara F, Parini D, Rizzo G (2020) Multidisciplinary Italian study group for STOmas (MISSTO). Enteral stoma care during the COVID-19 pandemic: practical advice. Colorectal Dis. 22(9):985-92

29. Mascagni D, Eberspacher C, Mascagni P, Arezzo A, Selvaggi F, Sturiale A et al (2020) From high volume to "zero" proctology: Italian experience in the COVID era. Int $\mathrm{J}$ Colorectal Dis 35(9): 1777-1780

30. George I, Salna M, Kobsa S, Deroo S, Kriegel J, Blitzer D et al (2020) The rapid transformation of cardiac surgery practice in the coronavirus disease 2019 (COVID-19) pandemic: insights and clinical strategies from a center at the epicenter. J Thorac Cardiovasc Surg 160(4):937-947.e2

31. Hussain A, Balmforth D, Yates M, Lopez-Marco A, Rathwell C, Lambourne $\mathrm{J}$ et al (2020) The pan London emergency cardiac surgery service: coordinating a response to the COVID-19 pandemic. J Card Surg 35(7):1563-1569

32. Griffin CL, Sharma V, Sarfati MR, Smith BK, Kraiss LW, McKellar SH et al (2020) Aortic disease in the time of COVID-19 and repercussions on patient care at an academic aortic center. J Vasc Surg 72(2):408-413

33. Hwang ES, Balch CM, Balch GC, Feldman SM, Golshan M, Grobmyer SR et al (2020) Surgical oncologists and the COVID19 pandemic: guiding cancer patients effectively through turbulence and change. Ann Surg Oncol 27(8):2600-2613

34. Goel SS, Greenbaum AB, Patel A, Little SH, Parikh R, Wyler von Ballmoos MC et al (2020) Role of teleproctoring in challenging and innovative structural interventions amid the COVID19 pandemic and beyond. JACC Cardiovasc Interv 13(16):1945-8

35. Dearani JA, Stephens EH, Guleserian KJ, Overman DM, Backer CL, Romano JC et al (2020) COVID-19: FAQs-congenital heart surgery recovery and defining a "new normal." World J Pediatr Congenit Heart Surg 11(5):548-556

36. Layfield E, Triantafillou V, Prasad A, Deng J, Shanti RM, Newman JG et al (2020) Telemedicine for head and neck ambulatory visits during COVID-19: evaluating usability and patient satisfaction. Head Neck 42(7):1681-1689

37. Lee AKF, Cho RHW, Lau EHL, Cheng HK, Wong EWY, Ku PKM et al (2020) Mitigation of head and neck cancer service disruption during COVID-19 in Hong Kong through telehealth and multi-institutional collaboration. Head Neck 42(7):1454-1459

38. Arrese M (2020) Telemedicine, COVID-19 and liver diseases: revamping remote care initiatives in hepatology. Ann Hepatol 19(4):339-340

39. Sherman CB, Said A, Kriss M, Potluri VS, Levitsky J, Reese PP et al (2020) In-person outreach and telemedicine in liver and intestinal transplant: a survey of national practices, impact of coronavirus disease 2019, and areas of opportunity. Liver Transpl 26(10): $1354-1358$

40. Fix OK, Serper M (2020) Telemedicine and telehepatology during the COVID-19 pandemic. Clin Liver Dis (Hoboken) 15(5): $187-190$

41. APASL Covid-19 Task Force, Lau G, Sharma M (2020) Clinical practice guidance for hepatology and liver transplant providers during the COVID-19 pandemic: APASL expert panel consensus recommendations. Hepatol Int 14(4):415-28

42. Kabaçam G, Dayangaç M, Üçbilek E, Erçin CN, Günsar F, Akyıldız $M$ et al (2020) The COVID-19 pandemic: clinical practice advice for gastroenterologists, hepatologists, and liver transplant specialists. Turk J Gastroenterol 31(5):348-355

43. Chao TN, Frost AS, Brody RM, Byrnes YM, Cannady SB, Luu $\mathrm{NN}$ et al (2021) Creation of an interactive virtual surgical rotation for undergraduate medical education during the COVID-19 pandemic. J Surg Educ 78(1):346-350

44. Dedeilia A, Sotiropoulos MG, Hanrahan JG, Janga D, Dedeilias P, Sideris M (2020) Medical and surgical education challenges and innovations in the COVID-19 era: a systematic review. In Vivo 34(3 Suppl):1603-1611

45. Salehi PP, Torabi SJ, Lee YH, Azizzadeh B (2020) Telemedicine practices of facial plastic and reconstructive surgeons in the United States: the effect of novel coronavirus-19. Facial Plast Surg Aesthet Med 22(6):464-470

46. Firriolo JM, Zeiderman MR, Sawyer SJ, Wong MS (2020) Advances in Surgical telemedicine during the coronavirus pandemic. Ann Plast Surg. 85(2S Suppl 2):S150

47. Reissis D, Georgiou A, Nikkhah D, O'Toole GUK (2020) Response to the COVID-19 pandemic: managing plastic surgery patients safely. Plast Reconstr Surg 146(2):250e-e251

48. Grome LJ, Reece E, Agrawal N, Kaplan J, Winocour S (2020) Protecting your reconstructive plastic surgery practice in a pandemic: five ways to mitigate this unique environment. Plast Reconstr Surg 146(2):254e 
49. Saad NH, AlQattan HT, Ochoa O, Chrysopoulo M (2020) Telemedicine and plastic and reconstructive surgery: lessons from the COVID-19 pandemic and directions for the future. Plast Reconstr Surg 146(5):680e-e683

50. Ashry AH, Alsawy MF (2020) Doctor-patient distancing: an early experience of telemedicine for postoperative neurosurgical care in the time of COVID-19. Egypt J Neurol Psychiatr Neurosurg 56(1):80

51. Ferraris KP, Golidtum JP, Zuñiga BKW, Bautista MCG, Alcazaren JC, Seng K et al (2020) Recapitulating the Bayesian framework for neurosurgical outpatient care and a cost-benefit analysis of telemedicine for socioeconomically disadvantaged patients in the Philippines during the pandemic. Neurosurg Focus 49(6): E14

52. Patel PD, Kelly KA, Reynolds RA, Turer RW, Salwi S, Rosenbloom ST et al (2020) Tracking the volume of neurosurgical care during the coronavirus disease 2019 pandemic. World Neurosurg 142:e183-e194

53. Eichberg DG, Basil GW, Di L, Shah AH, Luther EM, Lu VM et al (2020) Telemedicine in neurosurgery: lessons learned from a systematic review of the literature for the COVID-19 era and beyond. Neurosurgery 88(1):E1-12

54. Noureldine MHA, Pressman E, Greenberg MS, Agazzi S, van Loveren H, Alikhani P (2020) Letter to the editor "neurosurgical service coverage during the COVID-19 pandemic: the "battle plan' at the university of South of Florida affiliate hospitals." World Neurosurg 138:600-602

55. Mohanty A, Srinivasan VM, Burkhardt J-K, Johnson J, Patel AJ, Sheth SA et al (2020) Ambulatory neurosurgery in the COVID19 era: patient and provider satisfaction with telemedicine. Neurosurg Focus 49(6):E13

56. Bechstein M, Elsheikh S, Wodarg F, Taschner CA, Hanning U, Buhk J-H et al (2020) Interhospital teleproctoring of endovascular intracranial aneurysm treatment using a dedicated livestreaming technology: first experiences during the COVID-19 pandemic. BMJ Case Rep 13(10):e016722

57. Cho RHW, Yeung ZWC, Ho OYM, Lo JFW, Siu AKY, Kwan WMY et al (2020) Pearls of experience for safe and efficient hospital practices in otorhinolaryngology-head and neck surgery in Hong Kong during the 2019 novel coronavirus disease (COVID-19) pandemic. J Otolaryngol Head Neck Surg 49(1):30

58. Cronin AJ, Lopez JTJ, Pabla R (2020) Evaluation of remote OMFS assessments in the era of pandemic COVID-19 control measures. Br J Oral Maxillofac Surg 58(8):1023-1028

59. Hussaini AS, Clark CM, Patel AA, Russo ME, Chia SH, Davidson BJ et al (2020) Management of adult inpatient otolaryngologic consultations during the COVID-19 pandemic: a proposed tier-based triage system. Otolaryngol Head Neck Surg 163(2):330-334
60. Baird BJ, Sung CK (2020) Coronavirus disease-19: challenges associated with the treatment of head and neck oncology and laryngology patients in the coronavirus disease-19 era. Otolaryngol Clin North Am 53(6):1159-1170

61. Gupta PC, Kumar MP, Ram J (2020) COVID-19 pandemic from an ophthalmology point of view. Indian J Med Res 151(5):411-418

62. Koh A, Chen Y (2020) Perspective from Singapore and China on the COVID-19 pandemic: the new world order for ophthalmic practice. Ophthalmology 127(8):e49-50

63. Lin P-F, Naveed H, Eleftheriadou M, Purbrick R, Zarei Ghanavati M, Liu C (2021) Cataract service redesign in the postCOVID-19 era. Br J Ophthalmol 105(6):745-750

64. Langer PD, Bernardini FP (2020) Oculofacial plastic surgery and the COVID-19 pandemic: current reactions and implications for the future. Ophthalmology 127(9):e70-e71

65. Dunkerley S, Kurar L, Butler K, James M, Lowdon I (2020) The success of virtual clinics during COVID-19: a closed loop audit of the British orthopaedic association (BOAST) guidelines of outpatient orthopaedic fracture management. Injury 51(12):2822-2826

66. Parnes N, Tousant C, Perrine J, DeFranco MJ (2020) outpatient orthopedic rehabilitation in New York state during the COVID19 pandemic: therapist perspectives. Orthopedics 43(5):292-294

67. Kale NN, Patel AH, Leddy MJ, Savoie FH, Sherman WF (2020) The effect of COVID-19 on orthopedic practices and surgeons in Louisiana. Orthopedics 43(6):351-355

68. O'Reilly M, Merghani K, Bayer T (2020) Virtual assessment and management in foot and ankle surgery during the COVID-19 pandemic: an Irish experience. J Foot Ankle Surg 59(5):876

69. DeKeyser GJ, Brodke DS, Saltzman CL, Lawrence BD (2020) Response to the coronavirus disease 2019 pandemic by the spine division at a level-I academic referral center. J Am Acad Orthop Surg 28(24):1003-1008

70. Davey MS, Coveney E, Rowan F, Cassidy JT, Cleary MS (2020) Virtual fracture clinics in orthopaedic surgery: a systematic review of current evidence. Injury 51(12):2757-2762

71. Hiller SC, Dauw CA, Ghani KR (2020) Michigan urological surgery improvement collaborative. Kidney stone care and the COVID-19 pandemic: challenges and opportunities. J Urol 204(6):1122-4

72. Somani BK, Pietropaolo A, Coulter P, Smith J (2020) Delivery of urological services (telemedicine and urgent surgery) during COVID-19 lockdown: experience and lessons learnt from a university hospital in United Kingdom. Scott Med J 65(4):109-111

Publisher's Note Springer Nature remains neutral with regard to jurisdictional claims in published maps and institutional affiliations. 